(c) 2007 by the American Geophysical Union.

An edited version of this paper was published by $\underline{\mathrm{AGU}}$.

\title{
Importance of the sea surface curvature to interpret the normalized radar cross section
}

\author{
A. A. Mouche ${ }^{1,3}$, B. Chapron ${ }^{1}$, N. Reul ${ }^{1, *}$, D. Hauser ${ }^{2}$, Y. Quilfen ${ }^{1}$ \\ ${ }^{1}$ Laboratoire d'Océanographie Spatiale, Ifremer, Plouzané, France \\ ${ }^{2}$ Centre d'Etude des Environnements Terrestre et Planétaires, IPSL, CNRS/UVSQ, Vélizy, France \\ ${ }^{3}$ Also at Centre National d'Etudes Spatiales, Paris, France. \\ *: Corresponding author : N. Reul, email address : $\underline{\text { nreul@ifremer.fr }}$
}

\begin{abstract}
:
Asymptotic models (small perturbation and small slope approximation at first-order, Kirchhoff approximation or two-scale model) used to predict the normalized radar cross section of the sea surface generally fail to reproduce in detail backscatter radar measurements. In particular, the predicted polarization ratio versus incidence and azimuth angles is not in agreement with experimental data. This denotes the inability of these standard models to fully take into account the roughness properties with respect to the sensor's configuration of measurement (frequency, incidence, and polarization). On the basis of particular assumptions, to decompose the scattered electromagnetic field between zones covered with freely propagating waves and others where roughness and slopes are enhanced, recent works were able to match observations. In this paper, we do not assume such a decomposition but study the latest improvements obtained in the field of approximate scattering theories of random rough surfaces using the local and resonant curvature approximations. These models are based on an extension of the Kirchhoff Approximation up to first order to relate explicitly the curvature properties of the sea surface to the polarization strength of the scattered electromagnetic field. Consistency with previous approaches is discussed. As shown, dynamically taking into account the sea surface curvature properties of the surface is crucial to better interpret normalized radar crosssection and polarization ratio sensitivities to both sensor characteristics and geophysical environment conditions. The proposed developments, termed the Resonant Curvature Approximation (RCA), are found to reproduce experimental data versus incidence angle and azimuth direction. The polarization sensitivity to the wind direction and incidence angle is largely improved. Finally, Gaussian statistical assumption adopted to derive the analytical expression of the normalized radar cross section is also discussed. In particular, the third-order cumulant function is shown to better reproduce the secondorder up-/down-wind azimuth modulation. The proposed developments appear very promising for improvement of our understanding and analysis of both sea surface radar backscatter and Doppler signals.
\end{abstract}

Keywords: Resonant Curvature Approximation; sea surface radar; backscatter. 


\section{Introduction}

${ }_{36}$ As the capabilities of remote sensing instruments ever-increase, new opportunities to

${ }_{37}$ develop consistent inversion schemes of the sea surface geometry and kinematic appear.

${ }_{38}$ For instance, the high resolution SAR images are now commonly used to retrieve wind

39 fields thanks to the backscattered intensity power [Monaldo and Kerbaol, 2003] or the

40 sea surface velocity using the Doppler anomaly analysis [Chapron et al., 2003, 2005].

${ }_{41}$ Consequently, one could think about consistent inversion merging these two sources of

42 information. To resolve the remaining ambiguities in the normalized radar cross-section

${ }_{43}$ (NRCS) interpretation. In particular, it will help to better decipher between wind effects

${ }_{44}$ and current impacts on the apparent surface roughness.

${ }_{45}$ However, to date, large discrepancies between observations and model predictions still

46 remain. Asymptotic theories as the Small Slope Approximation (SSA) [Voronovich, 1994;

${ }_{47}$ Plant, 2002], Kirchhoff Approximation (KA), or more standard approaches as the Two-

${ }_{48}$ Scale Model (TSM) [Plant, 1986; Thompson, 1988; Romeiser et al., 1997] and the Small

49 Perturbation Method at first order (SPM-1) [Valenzuela, 1978] fail to correctly predict

50 the NRCS in both $\mathrm{HH}$ and VV co-polarizations under all environmental conditions and

${ }_{51}$ sensor configurations and characteristics. Data comparisons by several authors system-

${ }_{52}$ atically recall these weaknesses. The above cited models cannot reproduce the NRCS

${ }_{53}$ in both VV and $\mathrm{HH}$ polarizations with respect of incidence angle (see e.g. [Voronovich

${ }_{54}$ and Zavarotny, 2001; Kudryavtsev et al., 2003; Plant, 2003; Mouche et al., 2006b]). Data

${ }_{55}$ acquired simultaneously for both co-polarizations help to study more precisely the polar-

${ }_{56}$ ization ratio (PR) defined as the ratio of the NRCS in VV over the NRCS in HH. Mouche 
${ }_{57}$ et al. [2005] show that in C-band the PR is azimuth dependent with respect to the wind

${ }_{58}$ direction. In C-band, this dependency was clearly evidenced for incidence larger than $30^{\circ}$.

${ }_{59}$ Mouche et al. [2006b] show that the first order expansion of the Small Slope Approxima-

${ }_{6}$ tion (SSA-1) or TSM fail to reproduce this azimuth dependency. By construction SSA-1,

${ }_{61}$ SPM-1 and KA cannot reproduce any azimuthal variation for the PR. This is a strong

${ }_{62}$ limitation of such models. In opposite, TSM can lead to an azimuth dependency, but

${ }_{63}$ generally not in agreement with the data. This difference between TSM and other cited

${ }_{64}$ models clearly results from the efforts made in the TSM formalism to take into account

${ }_{65}$ the depolarization effects of the largest waves on the Bragg resonant waves (e.g. [Plant,

${ }_{66}$ 1986; Thompson, 1988; Romeiser et al., 1997; Valenzuela, 1978]). However, as often dis-

${ }_{67}$ cussed, TSM's formalism is somehow arbitrary to the choice of the parameter separating

${ }_{68}$ large modulating and small modulated waves. To overcome these issues and to propose

${ }_{69}$ consistent inversion schemes, one certainly need to advance in the field of approximate

70 theories of scattering from random rough surface [Elfouhaily and Guérin, 2004].

${ }_{71}$ In particular, Elfouhaily et al. [2003a] proposed a new asymptotic theory for wave

${ }_{72}$ scattering from rough surface taking into account the curvature effect of the surface on

${ }_{73}$ the scattered field. This curvature effect is a first order correction term of the zeroth

${ }_{74}$ order expression of the scattered field given by the Kirchhoff Approximation (KA). The

75 Local Curvature Approximation (LCA-1) formalism has the advantage to dynamically

76 reach both KA and the Small Perturbation Method at first order (SPM-1) depending

77 upon the surface properties. Mouche et al. [2006a] applied LCA-1 to the problem of

78 microwave scattering from ocean surface. Based on analytical comparisons with the Two-

79 Scale Model but also data comparisons, it was shown that LCA-1 polarization sensitivity 
so was very close to TSM and thus somehow inadequate to reproduce the NRCS of the ocean

${ }_{81}$ surface. But, in comparison with the TSM, LCA-1 solution is more general as it unifies

${ }_{82}$ dynamically SPM and KA asymptotic solutions and removes the issue concerning the

${ }_{83}$ dividing scale of the surface. Based on this analysis, a new asymptotic solution which

${ }_{84}$ provides a more realistic polarization sensitivity than LCA-1 has been proposed to restrict

${ }_{85}$ the curvature correction to the so-called resonant Bragg waves. This model, namely the

${ }_{86}$ Resonant Curvature Approximation (RCA), conserves the dynamical properties of LCA-

${ }_{87} 1$ to reach KA and SPM-1 asymptotic solution. Accordingly, the polarization ratio at a

s8 given incidence angle will be sea surface roughness dependant.

In this paper, we first expose the remaining issues in the field of the sea surface NRCS

90 prediction. Data and model comparisons are presented to illustrate our comments. Then,

${ }_{91}$ we briefly present the asymptotic solutions based on the extension of the KA to take into

${ }_{92}$ account the surface curvature effect of depolarization for the incident electromagnetic waves. Comparisons between the model and radar data help to discuss the importance of

${ }_{94}$ the sea curvature for backscattered signal interpretation. The issue about the statistical

${ }_{95}$ representation of the sea surface is also commented to resolve the up/down-wind asymme-

${ }_{96}$ try of the observed NRCS. Our conclusions and perspectives for the use of these models ${ }_{97}$ in the field of ocean remote sensing ends this work.

\section{Position of the problem}

To date, there is no electromagnetic model able to reproduce the NRCS in both VV and HH polarizations for all incidence angles, radar wavelength and wind conditions (speed, direction). In particular, the polarization sensitivity is not correctly reproduced. Figure 1 illustrates this point with two examples of $\mathrm{PR}$ in $\mathrm{Ku}$ and $\mathrm{C}$ band. The $\mathrm{PR}$ is defined 
as the ratio of the NRCS in VV over the NRCS in HH polarization. On figure 1 (a), we present the PR versus incidence angle calculated from Ku-band NRCS in both VV and HH polarizations measured by NSCAT. The data acquired in Ku-band were already presented by Quilfen et al. [1999]. We consider incidence angles from $20^{\circ}$ to $50^{\circ}$. $a_{0}^{p p}$ stands for the coefficient of the standard three-term Fourier model used for empirical formulations of the NRCS versus viewing angle with respect to wind direction:

$$
\sigma_{0}^{p p}(U, \theta, \Phi)=a_{0}^{p p}(U, \theta)+a_{1}^{p p}(U, \theta) \cos (\Phi)+a_{2}^{p p}(U, \theta) \cos (2 \Phi)
$$

${ }_{98}$ where $U$ is the near-surface wind speed, $\theta$ the radar's incidence angle and $\Phi$ the wind 99 direction relative to the radar's azimuth look direction. $p p$ denotes the co-polarization considered. With radar data, model predictions given by SPM-1, TSM, KA and SSA-1 
${ }_{114}$ largest incidence angles where there is a significant polarization difference between the ${ }_{115}$ backscattered signal in co-polarizations. SSA-1, by construction, imposes a polarization

116

117 


\section{Extended Kirchhoff model for the Normalized Radar Cross Section}

\subsection{Coordinates system and definitions}

To expose the general scattering problem, we adopt the same vectorial conventions than used by Elfouhaily and Guérin [2004] in their review on electromagnetic scattering theories. The right cartesian coordinate system is defined by the triplet of normalized vectors $(\hat{x}, \hat{y}, \hat{z})$, where the z-axis is directed upward. $\Sigma$ is the rough surface which separates the upper medium and the lower medium (respectively air and water in our specific case). The (sea) surface elevation is represented by $z=\eta(x, y)=\eta(\boldsymbol{r})$, where $\boldsymbol{r}$ is the horizontal component of the three-dimensional position wave vector $\boldsymbol{R}=(\boldsymbol{r}, z)$. According to these conventions, we consider a incident downward propagating electromagnetic plane wave with a wave-vector $\boldsymbol{K}_{0}=\left(\boldsymbol{k}_{0},-q_{0}\right)$. The up-going scattered waves is characterized by the wave-vector $\boldsymbol{K}=\left(\boldsymbol{k}, q_{k}\right) . \boldsymbol{k}_{0}$ and $\boldsymbol{k}$ are the horizontal components of the incident and scattered waves whereas $q_{0}$ and $q_{k}$ are the vertical ones. We define also $\boldsymbol{Q}_{h}$ and $Q_{z}$ related to the coordinates of the wave numbers $\boldsymbol{K}$ and $\boldsymbol{K}_{0}: \boldsymbol{Q}_{h}=\boldsymbol{k}-\boldsymbol{k}_{0}$ and $Q_{z}=q_{0}+q_{k}$.

The scattered field above and far away $(R \rightarrow \infty)$ from the sea surface is assumed to be related to the incident wave through the relation:

$$
\boldsymbol{E}_{s}(\vec{R})=-2 i \pi \frac{e^{i K R}}{R} \mathbb{S}\left(\boldsymbol{k}, \boldsymbol{k}_{0}\right) \cdot \hat{E}_{0}
$$

$\mathbb{S}\left(\boldsymbol{k}, \boldsymbol{k}_{0}\right)$ is the so-called scattering operator. $\boldsymbol{E}_{s}(\boldsymbol{R})$ and $\mathbb{S}\left(\boldsymbol{k}, \boldsymbol{k}_{0}\right)$ can be decomposed on the fundamental polarization basis:

$$
\boldsymbol{p}_{v}^{ \pm}( \pm k)=\frac{k \hat{z} \mp q_{k} \hat{k}}{K} \quad \boldsymbol{p}_{h}^{ \pm}( \pm k)=\hat{z} \times \hat{k}
$$

where the subscripts $v$ and $h$ indicate the vertical and horizontal polarizations, respectively. The minus superscript corresponds to the down-going plane waves while the plus 
superscript to the up-going waves. In this vectors basis, the scattering operator is related to the scattering amplitude $2 \times 2$ matrix through:

$$
\mathbb{S}\left(\boldsymbol{k}, \boldsymbol{k}_{0}\right)=\left[\begin{array}{l}
\boldsymbol{p}_{v}^{-}\left(\boldsymbol{k}_{0}\right) \\
\boldsymbol{p}_{h}^{-}\left(\boldsymbol{k}_{0}\right)
\end{array}\right]^{T} \cdot\left[\begin{array}{ll}
S_{v v}\left(\boldsymbol{k}, \boldsymbol{k}_{0}\right) & S_{v h}\left(\boldsymbol{k}, \boldsymbol{k}_{0}\right) \\
S_{h v}\left(\boldsymbol{k}, \boldsymbol{k}_{0}\right) & S_{h h}\left(\boldsymbol{k}, \boldsymbol{k}_{0}\right)
\end{array}\right] \cdot\left[\begin{array}{l}
\boldsymbol{p}_{v}^{+}(\boldsymbol{k}) \\
\boldsymbol{p}_{h}^{+}(\boldsymbol{k})
\end{array}\right],
$$
sion: solution.

$$
\sigma^{p q}=<\left|\mathbb{S}^{p q}\left(\boldsymbol{k}, \boldsymbol{k}_{0}\right)\right|^{2}>-\left|<\mathbb{S}^{p q}\left(\boldsymbol{k}, \boldsymbol{k}_{0}\right)>\right|^{2}
$$

\subsection{Local and Resonant Curvature Approximations}

Based on the work performed by Elfouhaily et al. [2003b], Elfouhaily et al. [2003a] expanded the scattering matrix up to the first order such as:

$$
\begin{aligned}
\mathbb{S}^{p q}\left(\boldsymbol{k}, \boldsymbol{k}_{0}\right) & =\frac{\mathbb{K}\left(\boldsymbol{k}, \boldsymbol{k}_{0}\right)}{Q_{z}} \int_{\boldsymbol{r}} e^{-i Q_{z} \eta(\boldsymbol{r})} e^{-i \boldsymbol{Q}_{H} \cdot \boldsymbol{r}} d \boldsymbol{r} \\
& -\mathrm{i} \int_{\boldsymbol{r}} \int_{\boldsymbol{\xi}} T\left(\boldsymbol{k}, \boldsymbol{k}_{0} ; \boldsymbol{\xi}\right) \hat{\eta}(\boldsymbol{\xi}) e^{-i Q_{z} \eta(\boldsymbol{r})} e^{-i\left(\boldsymbol{Q}_{H}-\boldsymbol{\xi}\right) \cdot \boldsymbol{r}} d \boldsymbol{\xi} d \boldsymbol{r}
\end{aligned}
$$

The scattering cross-section is given by the incoherent second order statistical expres-

$$
\mathbb{S}^{p q}\left(\boldsymbol{k}, \boldsymbol{k}_{0}\right)=\frac{1}{Q_{z}} \int_{\boldsymbol{r}} \mathbb{N}^{p q}\left(\boldsymbol{k}, \boldsymbol{k}_{0} ; \eta(\boldsymbol{r})\right) e^{-i Q_{z} \eta(\boldsymbol{r})} e^{-i \boldsymbol{Q}_{H} \cdot \boldsymbol{r}} d \boldsymbol{r},
$$

where $\mathbb{N}^{p q}\left(\boldsymbol{k}, \boldsymbol{k}_{0} ; \eta(\boldsymbol{r})\right)$ is a Kernel depending on the approach considered to establish the 
${ }_{156}$ is a kernel defined to take into account the surface curvature effects on the scattered field. $\mathbb{B}$ is the Bragg Kernel and $\mathbb{K}$ is the Kirchhoff Kernel (see e.g. Elfouhaily et al. [2003a] for

${ }_{158}$ their analytical expression). In Eq.(), the second term represents a first order correction

${ }_{159}$ to KA given by the first term. This first order curvature term has the property to reach dynamically both KA and SPM-1 limits with respect to the frequency and the properties

$T_{\text {rca }}\left(\boldsymbol{k}, \boldsymbol{k}_{0} ; \boldsymbol{\xi}\right)=\left[\mathbb{B}\left(\boldsymbol{k}, \boldsymbol{k}_{0} ; \boldsymbol{\xi}\right)-\mathbb{K}\left(\boldsymbol{k}, \boldsymbol{k}_{0}\right)\right] \delta\left(\boldsymbol{\xi}-\boldsymbol{Q}_{H}\right)$.

As already discussed by Mouche et al. [2006a], this solution can be compared with the improved Green's function method proposed by Shaw and Dougan [1998] excepted that the formulation helps to preserve the required shift and tilt invariance properties due to the LCA-1-like formalism of the RCA solution.

Assuming Gaussian statistics for the sea surface description, the derivation of the NRCS using the scattering matrix expansion up to the first order for any expansion such as $\mathbb{N}^{p q}\left(\boldsymbol{k}_{0}, \boldsymbol{k}\right)=\mathbb{N}_{0}^{p q}\left(\boldsymbol{k}_{0}, \boldsymbol{k}\right)+\int_{\boldsymbol{\xi}} N_{1}^{p q}\left(\boldsymbol{k}_{0}, \boldsymbol{k} ; \boldsymbol{\xi}\right) \hat{\eta}(\boldsymbol{\xi}) e^{i \boldsymbol{\xi} \cdot \boldsymbol{r}} d \boldsymbol{\xi}$ was already done and discussed in the context of LCA/RCA models by Mouche et al. [2006a]. In the case of microwave scattering from the sea surface sea surface, it was concluded that the predicted NRCS is very similar than the one using the phase perturbation method firstly proposed by Berman and Dacol [1990] and then applied by Voronovich and Zavarotny [2001] in the 
context of SSA-2. Thus, in this paper, for RCA, we consider that the first order term of the Volterra series in the scattering matrix expansion is a small perturbation in the phase term of the zeroth order contribution in Eq. () such as:

$$
\mathbb{S}\left(\boldsymbol{k}, \boldsymbol{k}_{0}\right)=\mathbb{K}_{0}\left(\boldsymbol{k}, \boldsymbol{k}_{0}\right) \int_{\boldsymbol{r}} e^{-i Q_{z} \eta(\boldsymbol{r})} e^{-i Q_{z} \delta_{\boldsymbol{k}, \boldsymbol{k}_{0}} \eta(\boldsymbol{r})} e^{-i \boldsymbol{Q}_{H} \cdot \boldsymbol{r}} d \boldsymbol{r}
$$

with

$$
\delta_{\boldsymbol{k}, \boldsymbol{k}_{0}} \eta(\boldsymbol{r})=\int_{\boldsymbol{\xi}} \frac{T_{\mathrm{lca} / \mathrm{rca}}\left(\boldsymbol{k}, \boldsymbol{k}_{0} ; \boldsymbol{\xi}\right)}{\mathbb{K}\left(\boldsymbol{k}, \boldsymbol{k}_{0}\right)} \hat{\eta}(\boldsymbol{\xi}) e^{i \boldsymbol{\xi} \cdot \boldsymbol{r}} d \boldsymbol{\xi} .
$$

${ }_{171} \tilde{\eta}(\boldsymbol{r})=\eta(\boldsymbol{r})+\delta_{\boldsymbol{k}, \boldsymbol{k}_{0}} \eta(\boldsymbol{r})$ can be seen as a modified surface elevation. In the case of RCA, 172 the first order curvature term is applied on the small resonant waves responsible of the ${ }_{173}$ Bragg scattering mechanism according to SPM-1 theory. Thus, the simplification of the ${ }_{174}$ scattering matrix expansion to describe the contribution of the curvature correction term through a phase modification is consistent with the small perturbation hypothesis.

Using the modified surface elevation for the statistical derivation of the NRCS, in the Kirchhoff integral, the characteristic function $<e^{\eta}>$ is replaced by $<e^{\tilde{\eta}}>$. Under Gaussian statistics, this formalism enables to have a tractable expression for the NRCS:

$$
\sigma_{0}^{p q}(\theta, \phi)=\left|\frac{\mathbb{K}\left(\boldsymbol{k}, \boldsymbol{k}_{0}\right)}{Q_{z}}\right|^{2} e^{-Q_{z}^{2} \tilde{\rho}(0)} \int_{\boldsymbol{r}}\left[e^{-Q_{z}^{2} \tilde{\rho}(\boldsymbol{r})}-1\right] e^{-i \boldsymbol{Q}_{H} \cdot \boldsymbol{r}} d \boldsymbol{r}
$$

with:

$$
\tilde{\rho}(\boldsymbol{r})=\int_{\boldsymbol{\xi}}\left|1+\frac{T_{\mathrm{lca} / \mathrm{rca}}\left(\boldsymbol{k}, \boldsymbol{k}_{0}, \boldsymbol{\xi}\right)}{\mathbb{K}\left(\boldsymbol{k}, \boldsymbol{k}_{0}\right)}\right|^{2} S(\boldsymbol{\xi}) e^{i \boldsymbol{\xi} \cdot \boldsymbol{r}} d \boldsymbol{\xi} .
$$

${ }_{176} \tilde{\rho}(\boldsymbol{r})$ is the so-called modified correlation function of a filtered spectrum and $S(\boldsymbol{\xi})$ the sea surface elevation spectrum. In the following, we use this formulation. 


\section{Results and Discussion}

\subsection{With Gaussian statistics for the sea surface description}

As a first comparison between data and model, we present the PR versus incidence angle given by RCA, LCA-1 and $\mathrm{KA}$ for a $10 \mathrm{~m} / \mathrm{s}$ wind speed in $\mathrm{C}$ - and $\mathrm{Ku}$-band in the case of an isotropic sea surface on figures 2 (a) and 2 (b). In both cases, we observe that the curvature correction term in RCA or LCA-1 ensures to the extended KA to get polarization sensitivity as the incidence increases. As expected, since the curvature correction term in RCA is restricted to the resonant Bragg waves, the induced polarization sensitivity is less than for LCA-1. From the data comparisons in $\mathrm{Ku}$ and $\mathrm{C}$ band presented here, we have a better agreement with RCA than with LCA-1. Figure 2 (c) presents the PR predicted by RCA and SSA-1 versus incidence angle for three frequencies. Focusing on the frequency dependency, we observe that the PR decreases when the frequency increases with both models. For SSA-1 (same comments can be done with KA or SPM-1) the only frequency dependency comes through the Kernel definition and is too small. In LCA-1 or RCA, the surface description controls the PR. This ensures, by construction, to reach dynamically both SPM-1 and KA asymptotic solutions, lead to KA results when $k_{0} \rightarrow \infty$ or when the perception of the surface by the sensor is flat (no curvature). Numerical computations of the PR show that the RCA solution is more frequency sensitive than SPM-1, SSA-1 or KA. This explains why the model agrees well with the data in both $\mathrm{Ku}$ and $\mathrm{C}$ bands on figures 2 (a) and $2(b)$.

As already mentioned above, another important feature in the backscattered signal for a given polarization, is the azimuth modulation with respect of the wind direction relative to the radar's azimuth look direction. From this modulation, we can infer the 
${ }_{222}$ wind direction relative to the radar's azimuth look direction. To be really complete with

${ }_{223}$ these data comparisons in C-band, we present on figure 3 (b) and 4 (b) the difference ${ }_{224}$ of NRCS $\sigma_{0}^{v v}-\sigma_{0}^{h h}$ in linear unit, DP hereafter, versus the wind direction relative to 225 the radar's azimuth look direction for the two wind speeds considered here. Once again, ${ }_{226} \mathrm{RCA}$ model is in better agreement with the data than other models. DP quantity is ${ }_{227}$ interesting since in literature, authors proposed to decompose the measured NRCS in a 228 polarized and a scalar part (e.g. [Quilfen et al., 1999]). Using DP quantity, we remove ${ }_{229}$ the scalar contribution to keep only the polarized part of the signal. In the case of ${ }_{230}$ backscattering, this part is taken into account through the first order curvature correction 
${ }_{267}$ the polarized contribution. This explains the observed UDA in the DP signal. Moreover,

${ }_{268}$ this implies that the opposite contribution of the UDA comes through the skewed form ${ }_{269}$ of the longer and the breaking waves which participate to the backscattering through

${ }_{270}$ the Kirchhoff mechanism. This idea consisting in associating the UDA of the breakers ${ }_{271}$ in a NRCS model was firstly applied by Kudryavtsev et al. [2003]. Same kind of dual ${ }_{272}$ co-polarizations analysis than the one performed by Chapron et al. [1997] or Quilfen et al. ${ }_{273}^{27}$ [1999] was proposed by Mouche et al. [2006b] in C-Band. This study based on STORM ${ }_{274}$ data for observations and Kudryavtsev et al. [2003] model also supports the idea of the ${ }_{275}$ breaking waves importance for the UDA through a scalar contribution to the NRCS.

Thus, we need to consider higher moments in the statistical derivation of the NRCS.

${ }_{277}$ The third order correction to the characteristic function enables to consider the skewness ${ }_{278}$ effect of the waves. In the local frame of RCA model, the modified characteristic function ${ }_{279}$ up to the third order is simply:

$<e^{j Q_{z}\left(\tilde{\eta}_{2}-\tilde{\eta}_{1}\right)}>\approx e^{-Q_{z}^{2}(\tilde{\rho}(0)-\tilde{\rho}(\boldsymbol{r}))} e^{i Q_{z}^{3} \tilde{S}_{\text {skew }}(\boldsymbol{r})}$

${ }_{280}$ where $\tilde{S}_{\text {skew }}$ is the skewness function associated to the modified surface height function.

${ }_{281}$ According to RCA formalism it is clear that any correcting order of the characteristic

${ }_{282}$ function can be polarization and frequency dependent. Following this idea, the NRCS ${ }_{283}$ when considering the skewness effect is:

$\sigma_{0}(\theta, \phi)=\left|\frac{\mathbb{K}\left(\boldsymbol{k}, \boldsymbol{k}_{0}\right)}{Q_{z}}\right|^{2} e^{-Q_{z}^{2} \tilde{\rho}(0)} \int_{\boldsymbol{r}}\left[e^{-Q_{z}^{2} \tilde{\rho}(\boldsymbol{r})+i Q_{z}^{3} \tilde{S}_{\mathrm{skew}}(\boldsymbol{r})}-1\right] e^{-i \boldsymbol{Q}_{H} \cdot \boldsymbol{r}} d \boldsymbol{r}$ 
${ }_{284}$ In this work we choose for the skewness function a generic formulation as proposed by

${ }_{225}$ Elfouhaily [1997] adjusted on surface slope skewness when $\boldsymbol{r} \rightarrow 0$ [Cox and Munk, 1954]:

$$
\begin{aligned}
& S_{\text {skew }}(\boldsymbol{r}) \underset{r \rightarrow 0}{=}-\frac{1}{6} x \sigma_{s x}\left(x^{2} \sigma_{s x}^{2} C_{03}+3 y^{2} \sigma_{s y}^{2} C_{21}\right) \approx-\frac{r^{3}}{6} \sigma_{s x}^{3} C_{03} \cos (\phi), \\
& S_{\text {skew }}^{\prime}(0)=S_{\text {skew }}^{\prime \prime}(0)=S_{\text {skew }}^{\prime \prime \prime}(0)=0
\end{aligned}
$$




\section{Conclusion}

308 
327 [2005] who proposed a semi-empirical model based on an explicit decomposition of the

${ }_{328}$ sea surface. In this model, the sea surface is separated in two parts. First, a regular one

${ }_{329}$ which is responsible for the specular reflection near nadir and for the Bragg scattering

330 of short modulated waves by longer one through a TSM (which means also a separation

331 of the scales for this regular part). Second, zones with enhanced roughness due to effect

332 of breaking waves on the sea surface which produce a scalar contribution to the NRCS

33 through specular reflection on theses steep slopes. Obviously a parallel between these

${ }_{334}$ approaches can be done as the zeroth order of RCA could be compared to the breaking

335 waves and longer waves contribution invoked by Kudryavtsev et al. [2003] and Kudryavtsev

336 et al. [2005] while the curvature correction term and the Bragg contribution could be

${ }_{337}$ associated to the same scattering process of the short resonant waves. Advantages of

${ }_{338} \mathrm{RCA}$ are of course the absence of any dividing parameter to separate scales of the sea

339 surface. Moreover, as the enhanced roughness zones contribution may be hard to precisely

340 parameterize, it could be convenient to use a model such as RCA which consider both

${ }_{341}$ contributions from regular and non-regular surface implicitely through the characteristic

${ }_{342}$ function. In a future work, an explicit comparison of these two models will be done. But

${ }_{343}$ as an important issue, it can be stated that PR modulations shall follow the roughness

344 distribution and disturbations.

${ }_{345}$ Finally, we discussed the implication of the non Gaussian statistics on the NRCS for the

346 sea surface description. Interestingly, it appears from RCA formalism that the charac-

${ }_{347}$ teristic function of the scattered field is polarization, incidence and frequency dependent.

${ }_{348}$ Moreover, RCA is in agreement with Chapron et al. [2003] conclusion about the UDA of

349 the Bragg waves and breaking waves at large incidence angle. Taking into account a third 
${ }_{350}$ order correction term in the characteristic function as a signature of breaking waves, it ap-

${ }_{351}$ pears that extraction of the third moment in the backscattered signal would be interesting

352 to improve our understanding of the impact of breaking events on the NRCS.

${ }_{353}$ A model such as RCA can be used to improve our understanding about the backscatter

354 signal modulations. As an example, since at large incidence angles the NRCS in HH ${ }_{355}$ is lower than the prediction of KA whereas it is the contrary for the VV polarization,

356 it can simply be concluded that the sensor is very sensitive to the small waves in VV

${ }_{357}$ polarization (more backscattered signal) whereas for $\mathrm{HH}$ polarization it is the contrary

${ }_{358}$ (less backscattered signal) which means that the sensor is more sensitive to the longer and

steeper waves in this configuration. At large incidence angles, such a large sensitivity to the

${ }_{360}$ longer and steeper waves than to the resonant Bragg waves will induces a larger Doppler

361 shift associated to the remote sensed waves than in VV polarization. Next, this model will

362 be used to derive an imaging radar model based on the correlation function modulation to

${ }_{363}$ interpret the variation of the NRCS due to changes in the geophysical parameters. This

364 will help to define a more consistent combined analysis between measured Doppler shifts

365 and backscatter signals.

${ }_{366}$ Acknowledgments. (Text here)

\section{Appendix A: NRCS expression of existing in the case of Gaussian statistics}




\section{A1. Small Perturbation Method at first order}

$$
\sigma_{0}^{B R}=\left|\mathbb{B}\left(\boldsymbol{k}, \boldsymbol{k}_{0}\right)\right|^{2} S\left(\boldsymbol{Q}_{H}\right)
$$

\section{A2. Two Scale Model}

$$
\sigma_{0}^{T S M}=\int_{-\infty}^{\infty} d(\tan \Psi) \int_{-\infty}^{\infty} d(\tan \delta) \sigma_{0}^{B R}\left(\theta_{i}\right) P(\tan \Psi, \tan \delta),
$$

376

377

where $P(\tan \Psi, \tan \delta)$ is the joint probability density of slopes for the long waves, $\theta_{i}$ the local angle, and $\sigma_{0_{B R}}$ the NRCS given by the SPM-1 due to the small roughness elements modulated by the longer waves. In our calculation this probability density is assumed Gaussian. The calculation of $\sigma_{0_{B R}}$ is done considering the angles corrections given by Elfouhaily et al. [1999] instead of initial Valenzuela's results [Valenzuela, 1978]:

$$
\theta_{i}=-\cos ^{-1}\left[\cos (\theta+\Psi) \cos \left(\tan ^{-1} \delta \cos \Psi\right)\right]
$$

with $S_{x}=\tan \Psi \quad$ and $\quad S_{y}=\tan \delta$,

1 the slopes of longer waves in and perpendicular to the incident plane. 
A3. Small Slope Approximation at first order

$$
\sigma_{0}^{S S A-1}=\left|\frac{\mathbb{B}\left(\boldsymbol{k}, \boldsymbol{k}_{0}\right)}{Q_{z}}\right|^{2} e^{-Q_{z}^{2} \rho(0)} \int_{\boldsymbol{r}}\left[e^{-Q_{z}^{2} \rho(\boldsymbol{r})}-1\right] e^{-i \boldsymbol{Q}_{H} \cdot \boldsymbol{r}} d \boldsymbol{r}
$$

A4. Kirchhoff Approximation

$$
\sigma_{0}^{K I R}=\left|\frac{\mathbb{K}\left(\boldsymbol{k}, \boldsymbol{k}_{0}\right)}{Q_{z}}\right|^{2} e^{-Q_{z}^{2} \rho(0)} \int_{\boldsymbol{r}}\left[e^{-Q_{z}^{2} \rho(\boldsymbol{r})}-1\right] e^{-i \boldsymbol{Q}_{H} \cdot \boldsymbol{r}} d \boldsymbol{r} .
$$




\section{References}

382 Bentamy, A., P. Queffelou, Y. Quilfen, and K. Katsaros, Ocean surface wind fields estimated from satellite active and passive microwave instruments, IEEE Trans. on Geosc. and Remote Sens., 37, 2469-2486, 1999.

Berman, D., and D. Dacol, Manifestly reciprocal scattering amplitudes for rough surfaces interface scattering, J. Acous. Soc. of Am., 87(5), 1990.

Chapron, B., V. Kerbaol, and D. Vandermark, A note on relationships between seasurface roughness and microwave polarimetric backscatter measurements: results form polrad'96, Proc. Int. Workshop POLRAD'96, pp. 55-64, 1997.

Chapron, B., F. Collard, and V. Kerbaol, Satellite synthetic aperture radar sea surface doppler measurements, Proc. of the 2nd Workshop on Coastal and Marine Applications of SAR, ESA SP-565, pp. 133-140, 2003.

Chapron, B., F. Collard, and F. Ardhuin, Direct measurements of ocean surface velocity from space: Interpretation and validation, J. Geophys. Res., 110(C07008), 2005.

Cox, C., and W. Munk, Measurements of the roughness of the sea surface from photogrphs of the sun's glitter, J. Opt. Soc., 44(11), 838-850, 1954.

Elfouhaily, T., A consistent wind and wave model and its application to microwave remote sensing of the ocean surface, Thesis dissertation. Denis Diderot university - Paris 7, France, 1997.

Elfouhaily, T., and C.-A. Guérin, A critical survey of approximate scattering wave theories from random rough surfaces, Waves In Rand. Media, 14(4), R1-R40, 2004.

Elfouhaily, T., B. Chapron, K. Katsaros, and D. Vandermark, A unified directionnal wave spectrum for long and short wind-driven waves, J. Geophys. Res., 102, 15,781-15,796, 
1997.

Elfouhaily, T., D. Thompson, D. Vandemark, and B. Chapron, A new bistatic model for electromagnetic scattering from perfectly conducting random surfaces, Waves In Rand. Media, 9, 281-294, 1999.

Elfouhaily, T., S. Guignard, R. Awdallah, and D. Thompson, Local and non-local curvature approximation: a new asymptottic theory for wave scattering, Waves In Rand. Media, 13, 321-337, 2003a.

Elfouhaily, T., M. Joelson, S. Guignard, and D. Thompson, Analytical comparison between the surface current integral equation and the second-order small slope approximation, Waves In Rand. Media, 13, 165-176, 2003b.

Hauser, D., P. Dubois, and G.Caudal, Polarimetric wind-scatterometer measurements during polrad'96, Proc. of Int. Workshop POLRAD'96, pp. 55-64, 1997.

Hauser, D., T. Podvin, M. Dechambre, R. Valentin, G. Caudal, and J.-F. Daloze, Storm: A new polarimetric real aperture radar for earth observations, Proc. of ESA POLinsar Int. Workshop, 2003.

Herbasch, H., Cmod5 an improved geophysical model function for ers scatterometry, ECMWF, Internal Report, 2003.

Kudryavtsev, V., D. Hauser, G. Caudal, and B. Chapron, A semiempirical model of the normalized radar cross-section of the sea surface: 1. background model, J. Geophys. Res., 108(C3), 2003.

Kudryavtsev, V., D. Akimov, J. Johannessen, and B. Chapron, On radar imaging of current features: 1. model and comparison with observations, J. Geophys. Res., 110(C07016), 2005. 
${ }_{427}$ Monaldo, F., and V. Kerbaol, The sar measurement of ocean surface winds: An overview,

Proc. of the 2nd Workshop on Coastal and Marine Applications of SAR, ESA SP-565, pp. 15-32, 2003.

Mouche, A., D. Hauser, J.-F. Daloze, and C. Guérin, Dual-polarization measurements at c-band over the ocean: Results from ariborne radar observations and comparison with envisat asar data, IEEE Trans. on Geosc. and Remote Sensing, 43(4), 753-769, 2005.

Mouche, A., B. Chapron, and N. Reul, A simplified asymptotic theory for ocean surface electromagnetic waves scattering, Submitted to WRM, 2006a.

Mouche, A., D. Hauser, and V. Kudryavtsev, Radar scattering of the ocean surface and sea-roughness properties: A combined analysis from dual-polarizations airborne radar observations and models in c band, J. Geophys. Res., 111(C09004), 2006b.

Plant, W., A two-scale model of short wind-generated waves and scatterometry, J. Geophys. Res., 91(C9), 10,735-10,749, 1986.

Plant, W., A stochastic, multiscale model of microwave backscatter from the ocean, $J$. Geophys. Res., 107(C9), 2002.

Plant, W., Microwave sea return at moderate to high incidence angles, Waves In Rand. Media, 9, 339-354, 2003.

Quilfen, Y., B. Chapron, A. Bentamy, J. Gourrion, T. Elfouhaily, and D. Vandermark, Global ers-1 and 2 nscat observations: Upwind/crosswind and upwind/downwind measurements, J. Geophys. Res., 104(C5), 11,459-11,469, 1999.

Romeiser, R., W. Alpers, and V. Wismann, An improved composite surface model for the radar backscattering cross-section of the ocean surface: 1. theory of the model and optimization/validation by scatterometer data, J. Geophys. Res., 102(C11), 25,237- 

$25,250,1997$.

Shaw, W., and A. Dougan, Green's function refinement as an approach to radar backscatter: General theory and application to lga scattering from the ocean, IEEE Transaction on Antenna Propagation, 46(1), 57-66, 1998.

Stoffelen, A., and D. Anderson, Scatterometer data interpretation: Estimation and validation of the transfer function cmod4, J. Geophys. Res., 102, 5767-5780, 1997.

Thompson, D., Calculation of radar backscatter modulations from internal waves, J. Geophys. Res., 93(C10), 12,371-12,380, 1988.

Valenzuela, G., Theories for the interactions of electromagnetic and oceanic waves - a review, Boundary-Layer Met., 13, 61-85, 1978.

so Voronovich, A., Small-slope approximation for electromagnetic wave scattering at a rough interface of two dielectric half-spaces, Waves In Rand. Media, 4, 337-367, 1994.

${ }_{22}$ Voronovich, A., and V. Zavarotny, Theoritical model for scattering of radar signals in ku${ }_{463}$ and c-bands from a rough sea surface with breaking waves, Waves In Rand. Media, 11, 247-269, 2001. 
(a)

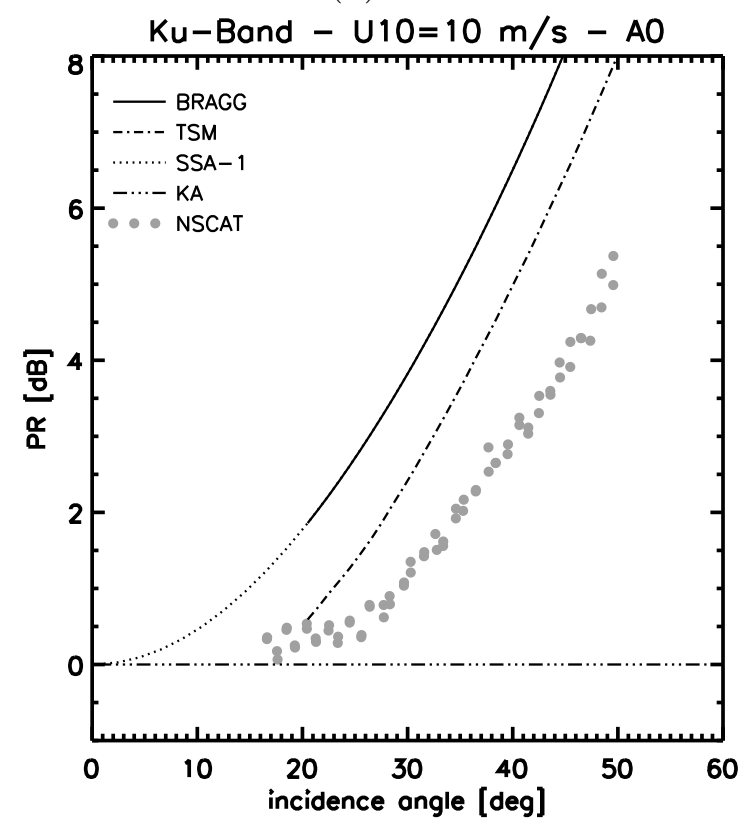

(b)

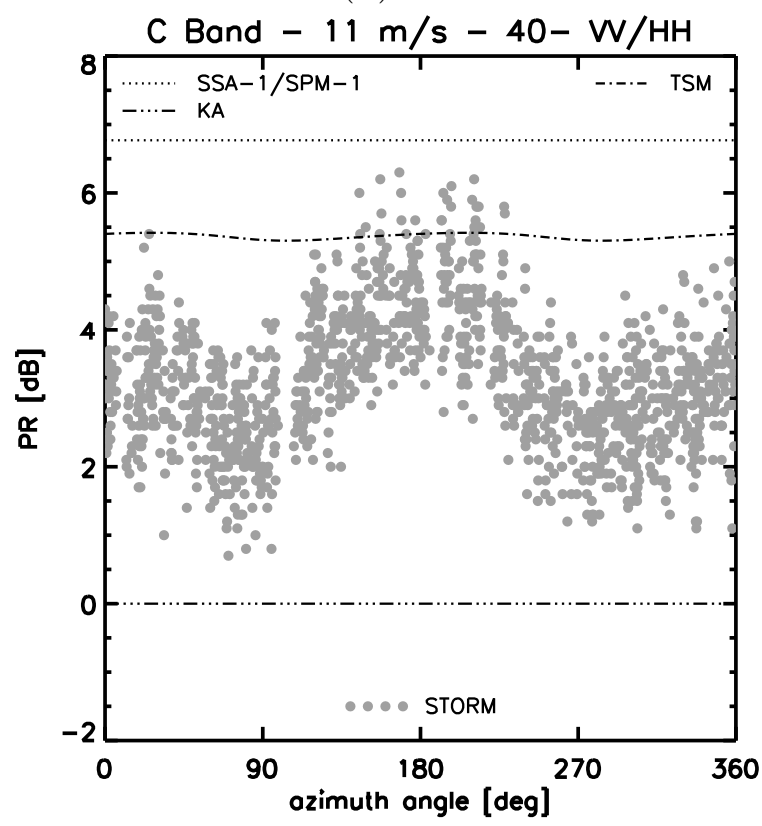

Figure 1. (a) Polarization ratio versus incidence angle in $\mathrm{Ku}$ band for a $10 \mathrm{~m} / \mathrm{s}$ ten meters high wind speed in the case of an isotropic sea surface. (b) Polarization ratio versus wind direction relative to the radar's azimuth look direction for a $11 \mathrm{~m} / \mathrm{s}$ ten meters high wind speed and a $40^{\circ}$ incidence angle. 
(a)

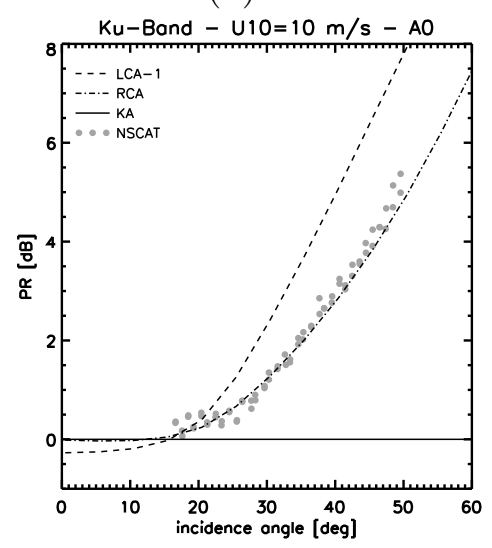

(b)

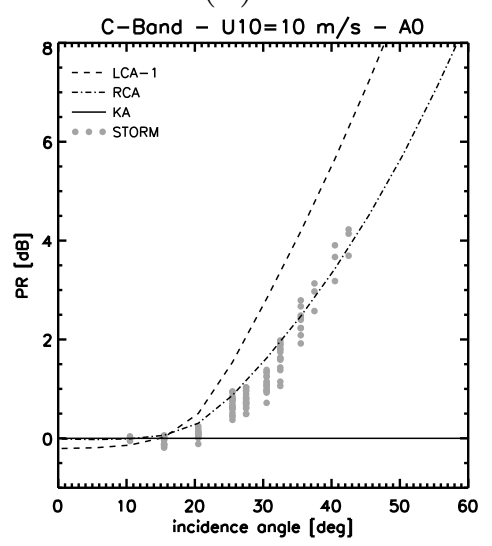

(c)

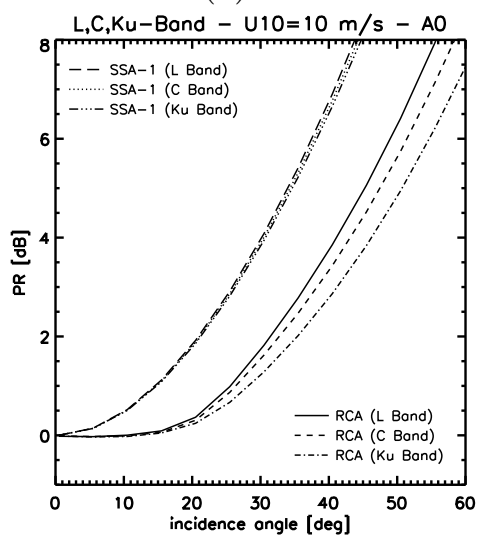

Figure 2. (a) Polarization ratio versus incidence angle in $\mathrm{Ku}$ band for a $10 \mathrm{~m} / \mathrm{s}$ ten meters high wind speed in the case of an isotropic sea surface. Solid, dashed and dashed-dotted lines are respectively the predictions given by KA, LCA and RCA models. Data are from NSCAT. (b) Same than (a) but for C-Band. Data are from STORM radar. (c) Polarization ratio versus incidence angle for a $10 \mathrm{~m} / \mathrm{s}$ ten meters high wind speed in the case of an isotropic sea surface in $\mathrm{Ku}, \mathrm{C}$ and $\mathrm{L}$ Band given by RCA and SSA-1. 
(a)

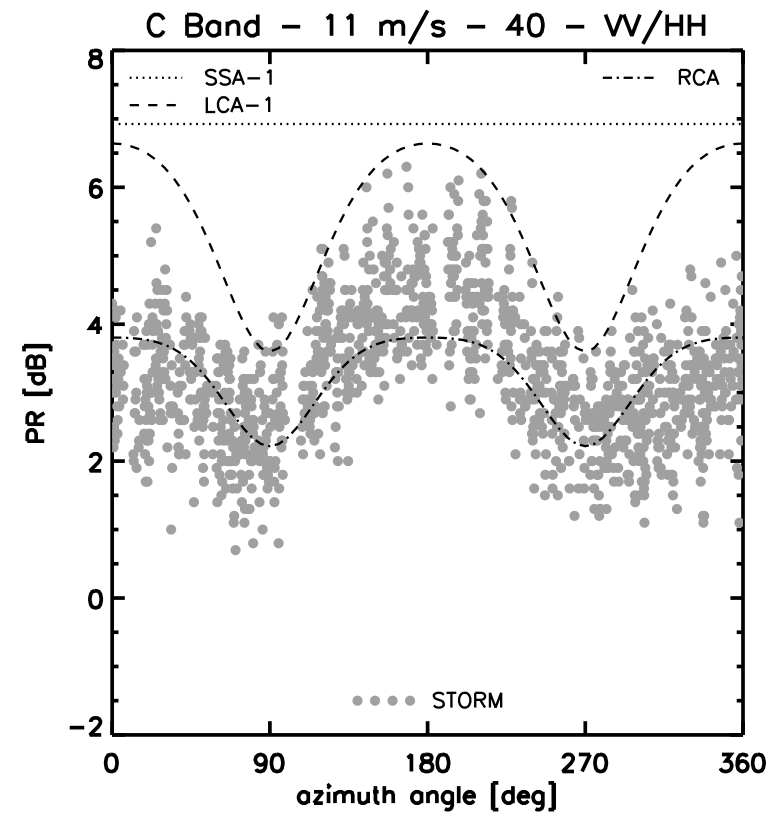

(c)

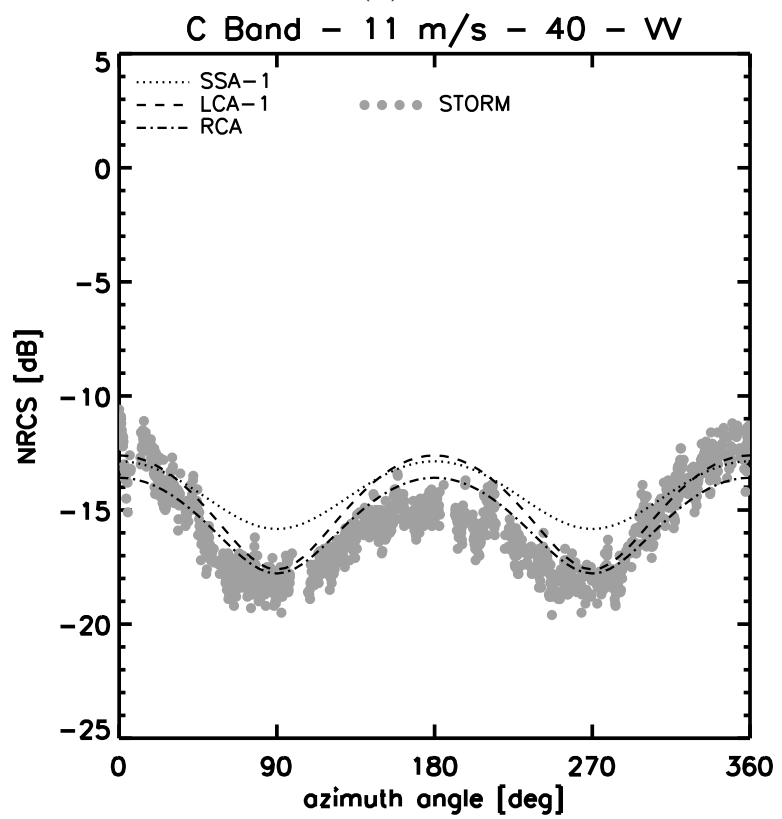

(b)

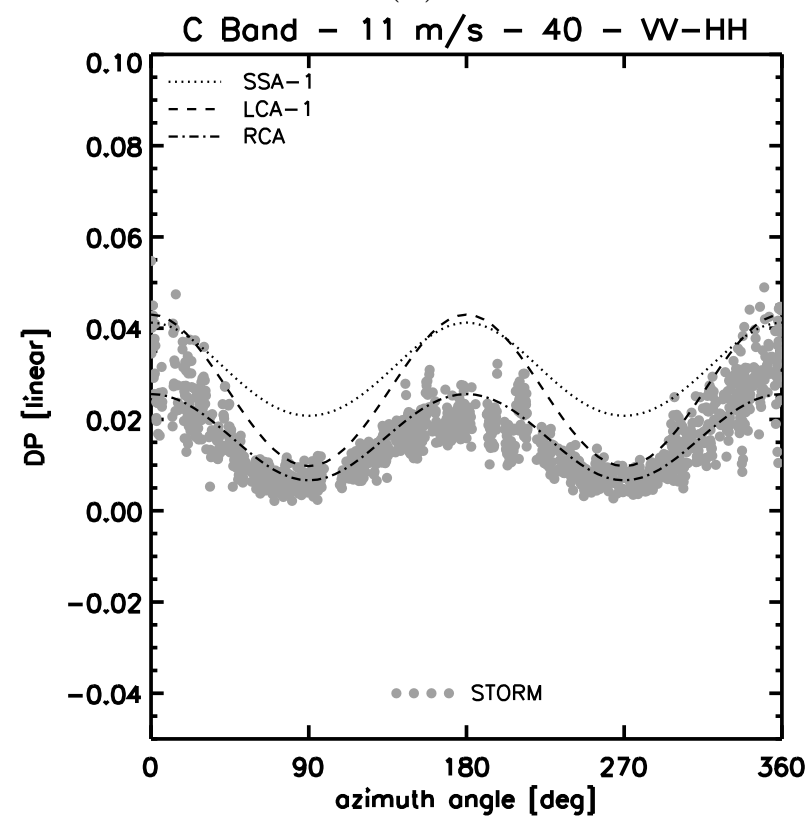

(d)

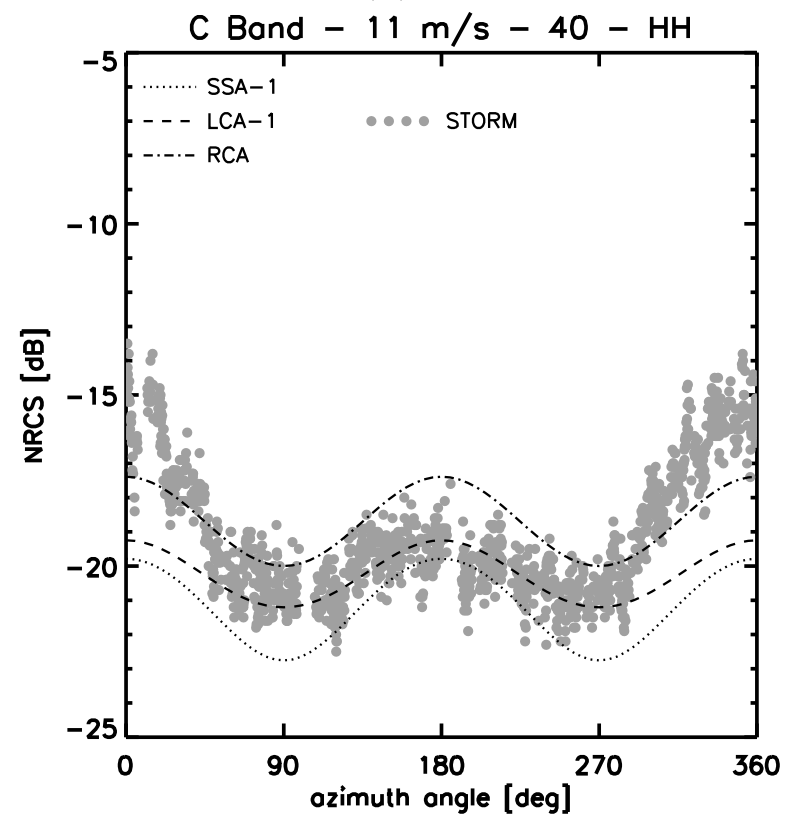

Figure 3. (a) Polarization ratio versus wind direction relative to the radar's azimuth look direction for a $11 \mathrm{~m} / \mathrm{s}$ ten meters high wind speed, a $40^{\circ}$ incidence angle in $\mathrm{C}$ band. (b) Same but for the difference of NRCS. (c) Same but for the NRCS in VV polarization. (d) Same but for the NRCS in HH polarization. 
(a)

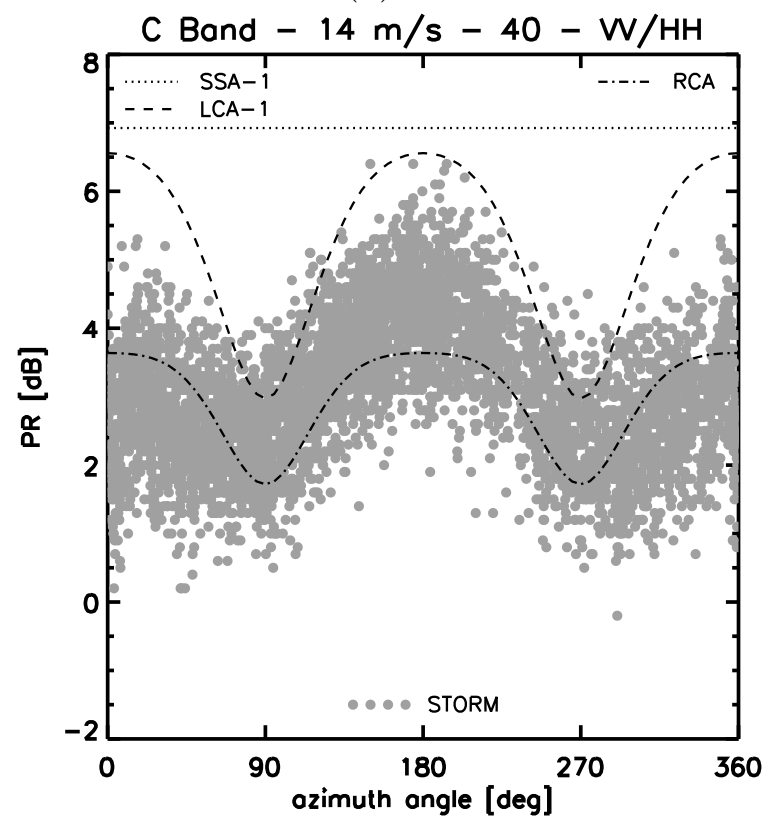

(c)

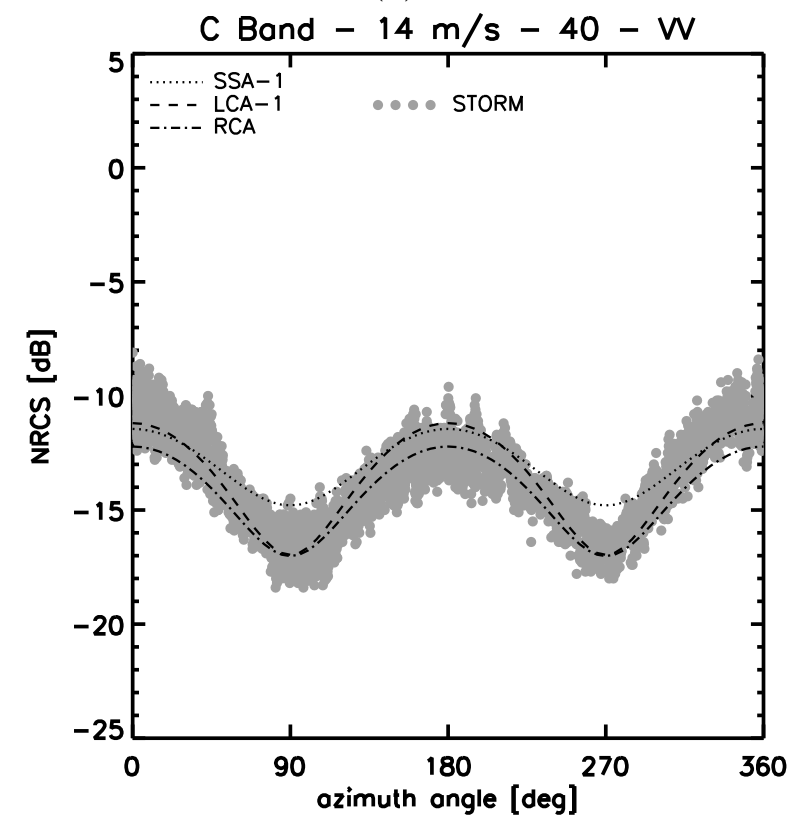

(b)

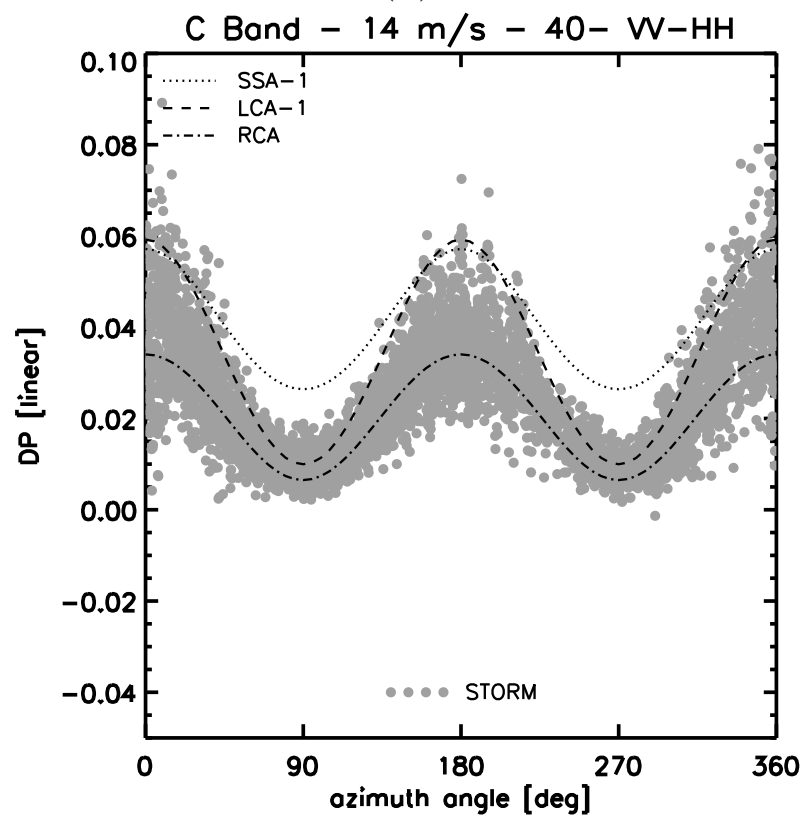

(d)

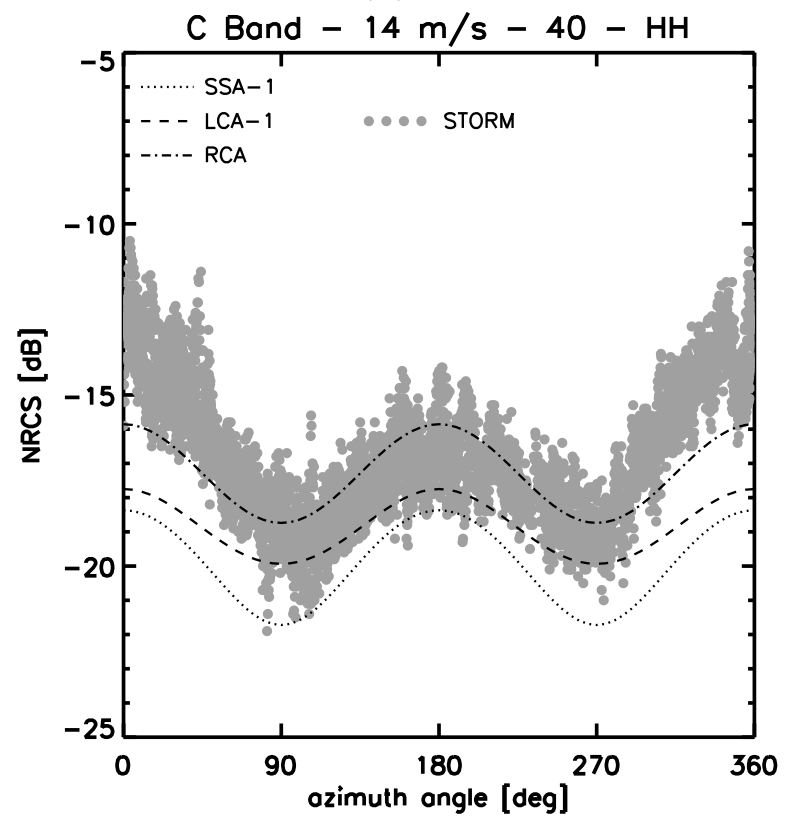

Figure 4. (a) Polarization ratio versus wind direction relative to the radar's azimuth look direction for a $14 \mathrm{~m} / \mathrm{s}$ ten meters high wind speed, a $40^{\circ}$ incidence angle in $\mathrm{C}$ band. (b) Same but for the difference of NRCS. (c) Same but for the NRCS in VV polarization. (d) Same but for the NRCS in HH polarization. 
(a)

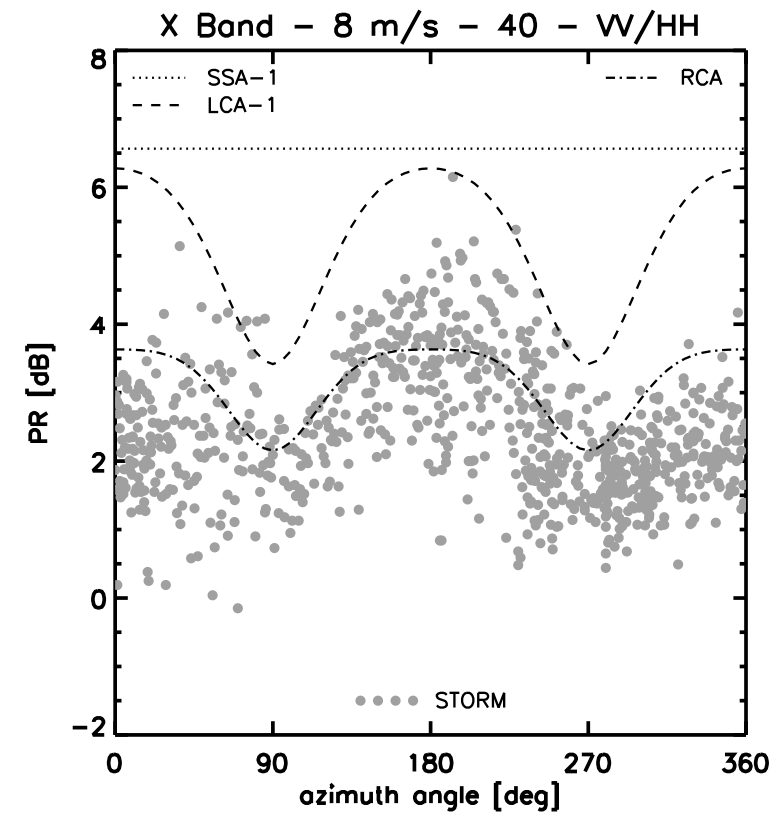

(c)

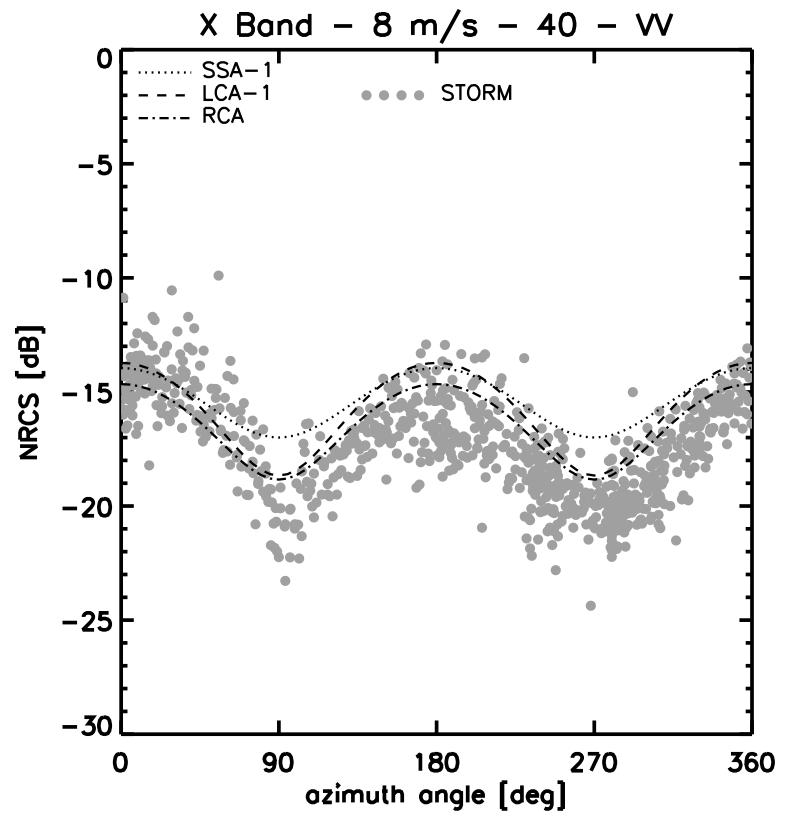

(b)

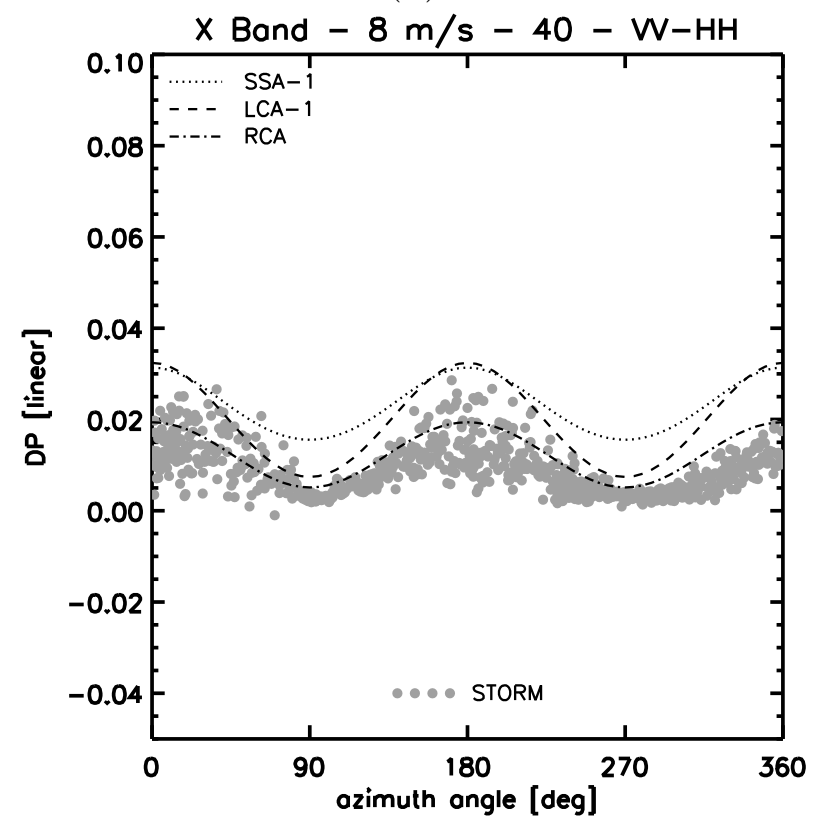

(d)

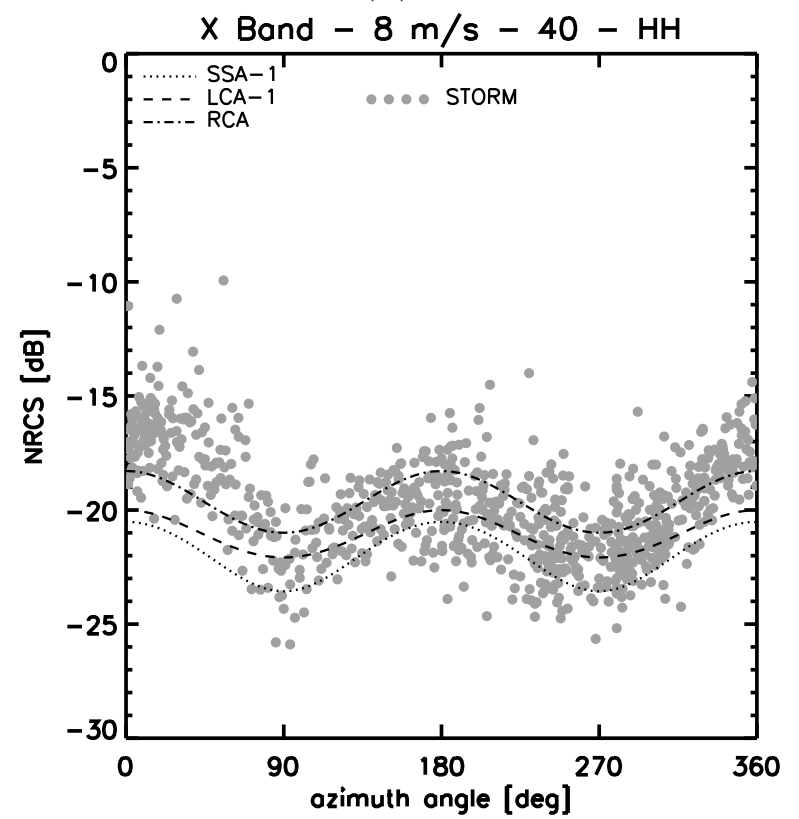

Figure 5. (a) Polarization ratio versus wind direction relative to the radar's azimuth look direction for a $8 \mathrm{~m} / \mathrm{s}$ ten meters high wind speed, a $40^{\circ}$ incidence angle in $\mathrm{X}$ band. (b) Same but for the difference of NRCS. (c) Same but for the NRCS in VV polarization. (d) Same but for the NRCS in HH polarization. 
(a)

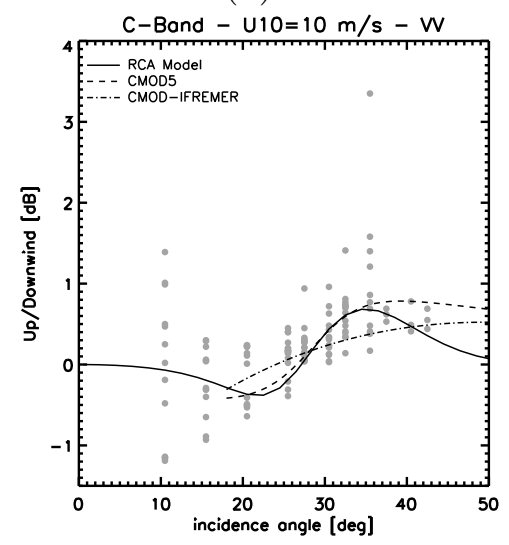

(d)

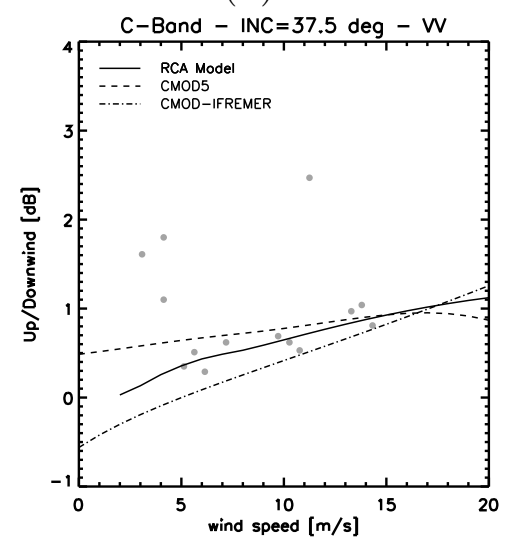

(b)

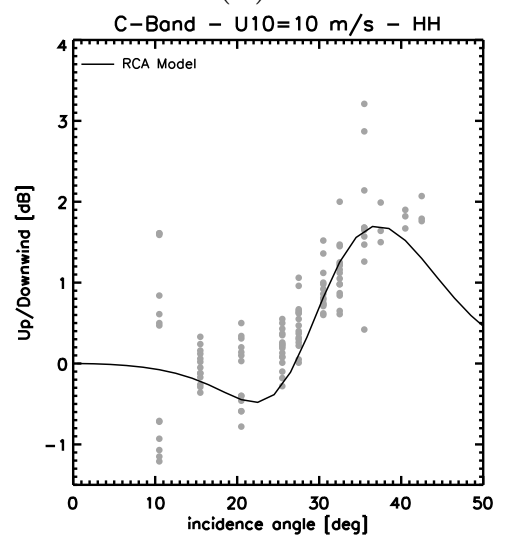

(e)

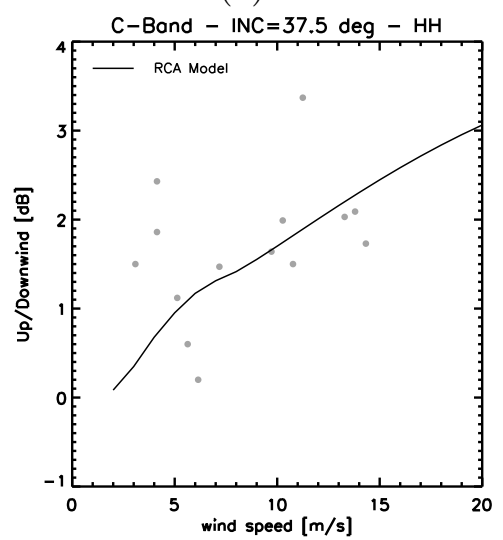

(c)

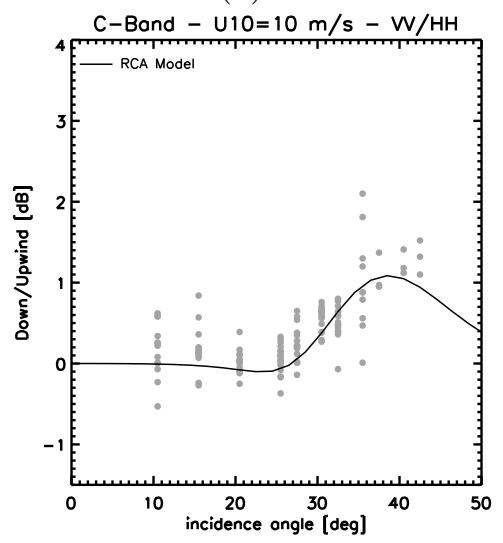

(f)

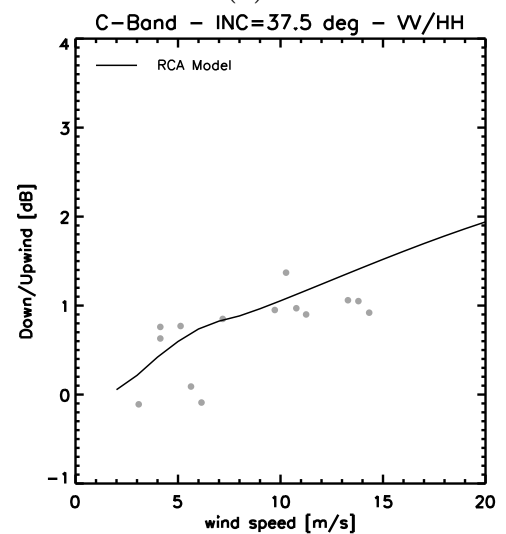

Figure 6. Top panel: UDA versus incidence angle in (a) VV and (b) HH polarizations for a $10 \mathrm{~m} / \mathrm{s}$ ten meters high wind speed. (c) Downwind to upwind asymmetry of the PR versus incidence angle for a $10 \mathrm{~m} / \mathrm{s}$ ten meters high wind speed. Bottom panel: UDA versus incidence angle for (a) VV and (b) HH polarizations for a 37.5 incidence angle. (c) Downwind to upwind asymmetry of the PR versus incidence angle for a $37.5^{\circ}$ incidence angle 
(a)

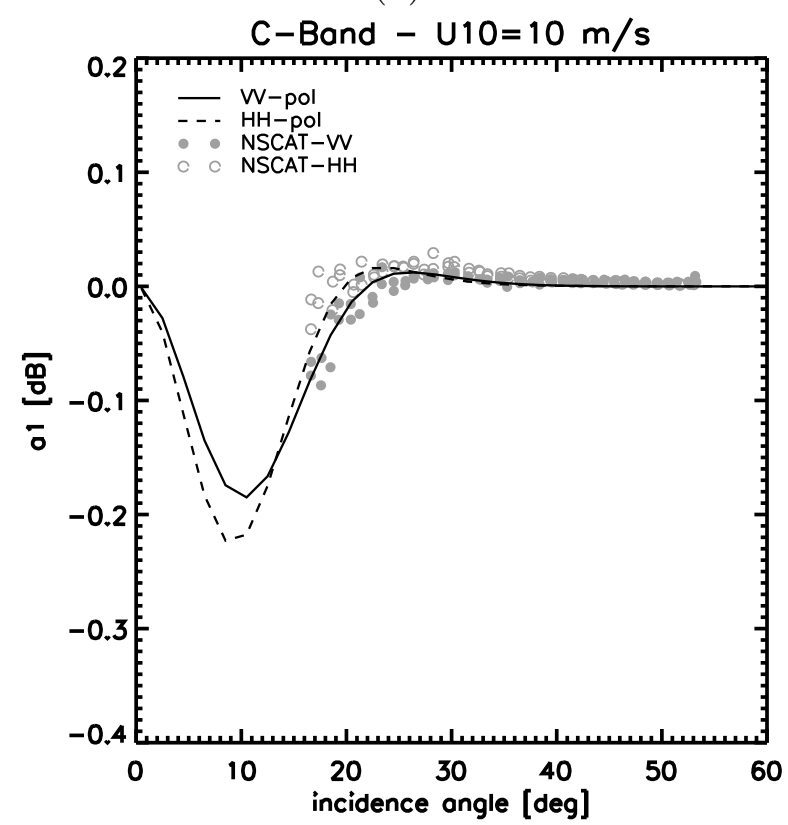

(b)

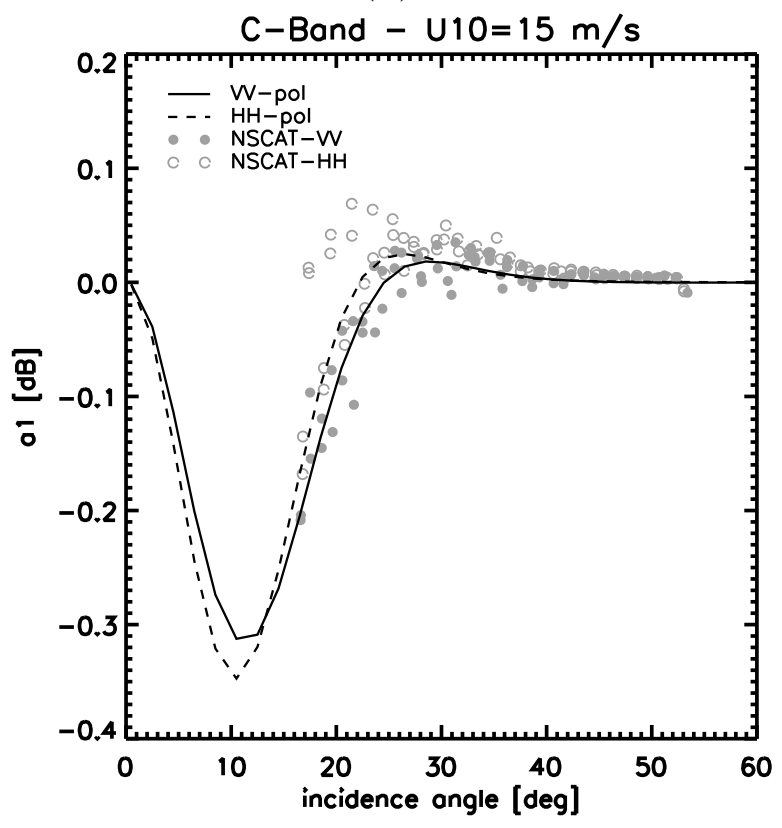

Figure 7. $\quad a_{1}^{v v}$ and $a_{1}^{h h}$ coefficients as a function of the incidence angle in Ku-Band for two given ten meters high wind speeds: (a) $10 \mathrm{~m} / \mathrm{s}$ and (b) $10 \mathrm{~m} / \mathrm{s}$. 
(a)

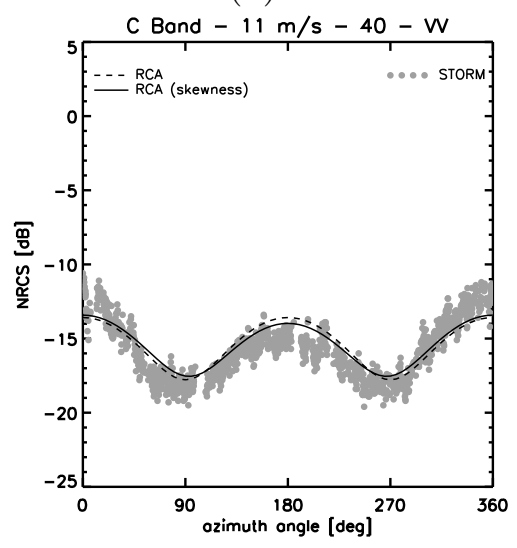

(d)

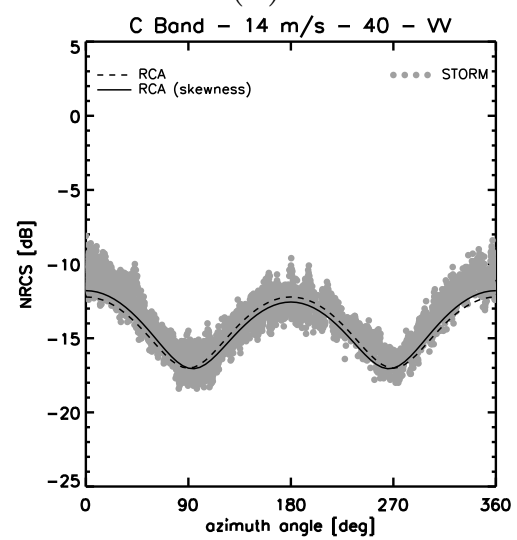

(b)

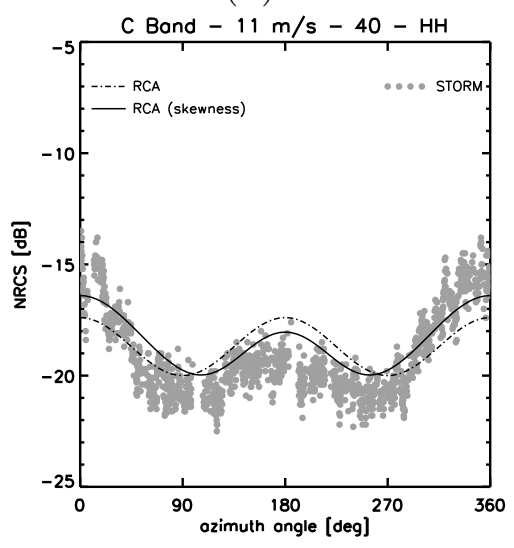

(e)

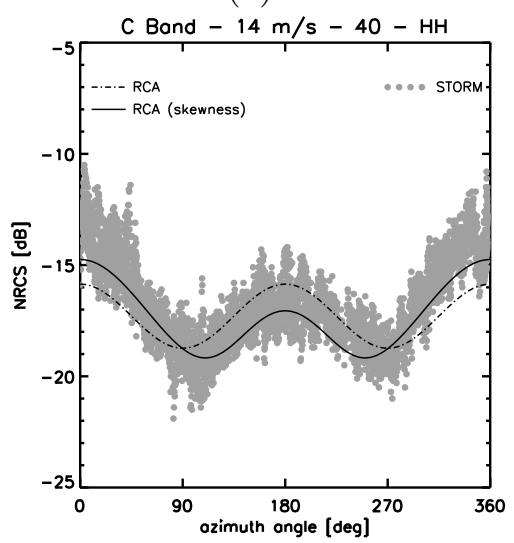

(c)

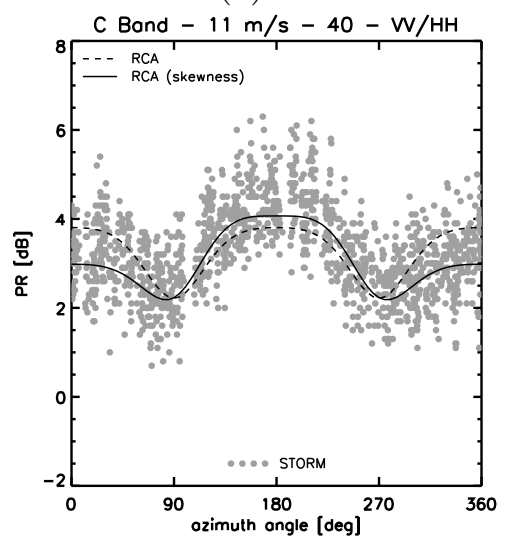

(f)

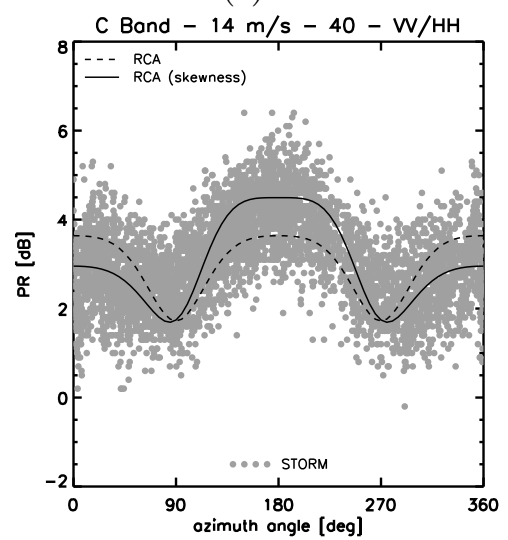

Figure 8. Top panel: NRCS versus wind direction relative to the radar's azimuth look direction for a $11 \mathrm{~m} / \mathrm{s}$ ten meters high wind speed, a $40^{\circ}$ incidence angle in $\mathrm{C}$ band in (a) $\mathrm{VV}$ and (b) $\mathrm{HH}$ polarizations. (c) Same for the PR. Bottom panel: NRCS versus wind direction relative to the radar's azimuth look direction for a $14 \mathrm{~m} / \mathrm{s}$ ten meters high wind speed, a $40^{\circ}$ incidence angle in $\mathrm{C}$ band in (a) VV and (b) HH polarizations. (c) Same for the PR. 